\title{
Improved V-Leach Protocol in Wireless Sensor Network with Data Security .
}

\author{
Hiral Shah ${ }^{1}$, S. R. Bhoyar ${ }^{2}$ \\ ${ }^{\text {I }}$ Student of Department of Electronics \& Telecommunication, Rajiv Gandhi college of Engg., Mumbai \\ University, India) \\ ${ }^{2}$ (Assistant Professorof Department of Electronics \& Telecommunication, Rajiv Gandhi college of Engg., \\ Mumbai University, India)
}

\begin{abstract}
Wireless sensor network requires robust and energy efficient communication protocols to minimize the energy consumption and increase the security as much as possible. The researchers mostly they are considering energy consuming. A cluster-based scheme is proposed as a solution for this issue. The suggested scheme extends Improved V-Leach algorithm and enables multi-hop transmissions among the clusters by incorporating the selection of cooperative sending and receiving nodes. The functioning of the suggested system is evaluated in terms of reliability and energy efficiency. Simulation results show that tremendous energy savings can be achieved by adopting hard network lifetime scheme among the clusters and of security and overall throughput.
\end{abstract}

Keywords: Clustering, Wireless sensor network, cluster head,Protocols

\section{Introduction}

A wireless sensor network (WSN) consists of sensor nodes capable of collecting information from the environment and communicating with each other via wireless transceivers. The collected data will be delivered to one or more sinks, generally via multihop communication[1]. The sensor nodes are typically expected to operate with batteries and are often deployed to not-easily-accessible or hostile environment, sometimes in large quantities. It can be difficult or impossible to replace the batteries of the sensor nodes. On the other hand, the sink is typically rich in energy. Since the sensor energy is the most precious resource in the WSN, efficient utilization of the energy to prolong the network lifetime has been the focus of much of the research on the WSN. The communications in the WSN has the many-to-one property in that data from a large number of sensor nodes tend to be concentrated into a few sinks. Since multi-hop routing is gener/ ally needed for distant sensor nodes from the sinks to save energy, the nodes near a sink can be burdened with relaying a large amount of traffic from other nodes. Sensor nodes are resource constrained in term of energy, processor and memory and low range communication and bandwidth. Limited battery power is used to operate the sensor nodes and is very difficult to replace or recharge it, when the nodes die. This will affect the network performance. Energy conservation increase lifetime of the network. Optimize the communication range and minimize the energy usage, we need to conserve the energy of sensor nodes .Sensor nodes are deployed to gather information and desired that all the nodes works continuously and transmit information as long as possible. This makes lifetime issue in wireless sensor networks. Sensor nodes spend their energy during transmitting the data, receiving and relaying packets. Hence, designing routing algorithms that maximize the life time until the first battery expires is an important consideration. Designing energy aware algorithm increases lifetime of sensor nodes. In some applications the network size is larger required scalable architectures. Energy conservation in wireless sensor networks has been the primary objective, but however, this constrain is not the only consideration for efficient working of wireless sensor networks. There are other objectives like scalable architecture, routing and latency. In most of the applications of wireless sensor networks are envisioned to handled critical scenarios where data retrieval time is critical, i.e., delivering information of each individual node as fast as possible to the base station become an important issue. It is important to guarantee that information can be successfully received to the base station the first time instead of being retransmitted. In wireless sensor network data gathering and routing are challenging tasks due to their dynamic and unique properties. Many routing protocols are developed, but among those protocols cluster based routing protocols are energy efficient, scalable and prolong the network lifetime. In the event detection environment nodes are idle most of the time and active at the time when the event occur. Sensor nodes periodically send the gather information to the base station[2]. Routing is an important issue in data gathering sensor network, while on the other hand sleep-wake synchronization are the key issues for event detection sensor networks. Low Energy Adaptive Clustering Hierarchy (LEACH) is the first hierarchical cluster-based routing protocol for wireless sensor network which partitions the nodes into clusters, in each cluster a dedicated node with extra privileges called Cluster Head $(\mathrm{CH})$ is responsible for creating and manipulating a TDMA (Time division multiple access) schedule and sending aggregated data from nodes to the 
BS where these data is needed using CDMA (Code division multiple access). Remaining nodes are cluster members. nodes if it will become a $\mathrm{CH}$ or not. This decision takes into account when the node served as a $\mathrm{CH}$ for the last time (the node that hasn't been a $\mathrm{CH}$ for long time is more likely to elect itself than nodes that have been a $\mathrm{CH}$ recently). In the following advertisement phase, the $\mathrm{CHs}$ inform their neighborhood with an advertisement packet that they become $\mathrm{CHs}$. Non- $\mathrm{CH}$ nodes pick the advertisement packet with the strongest received signal strength. In the next cluster setup phase, the member nodes inform the $\mathrm{CH}$ that they become a member to that cluster with "join packet" contains their IDs using CSMA. After the cluster-setup sub phase, the $\mathrm{CH}$ knows the number of member nodes and their IDs. Based on all messages received within the cluster, the CH creates a TDMA schedule, pick a CSMA code randomly, and broadcast the TDMA table to cluster members. After that steady-state phase begins[3]. A new version of LEACH called Improved V leach is proposed to add security to this energy conservation system.

\section{Modules}

To reduce the work load, we are dividing our project into small modules, they are given as bellow.

1. Grid sensor network deployment

2. Cluster formation

a. Collecting grid information

b. Energy calculation

3. Cluster head changes

4. Secure data transmission

\section{Grid sensor network deployment:} deployment.

In sensor network, we can deploy the sensor in two types; one is random deployment and optimal grid

Whenever we used random deployment, the network will get many of the problems like as coverage problem, energy reduction problem and interferences. To avoid this many problems we are going to use optimal grid sensor deployment.

Grid sensor network deployment is nothing but deployment of sensor in fixed and optimally calculated senor placement in certain distance.

Using grid sensor deployment we can improve the coverage area and also we can avoid the high cost.
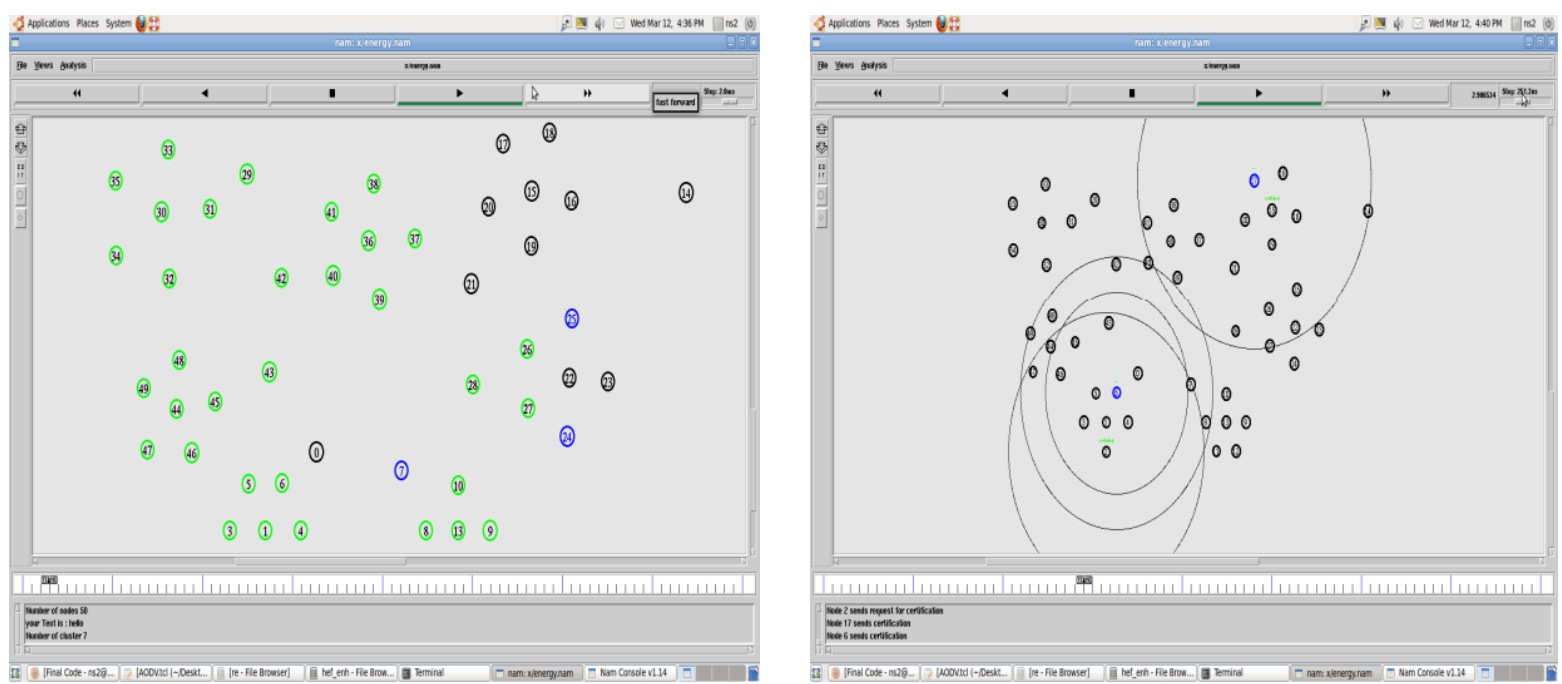

Fig 1. Network Animator showing formation of Clusters and Cluster Head Selection.

\section{Cluster formation}

Cluster formation is the process of grouping sensor devices by collecting the grid location information and gathering energy information.

In our project, we are using the grid sensor deployment, so we are defining cluster formation will be done within the grid. So initially we are calculating the each and every node location information and energy of the node. We are initializing the network with same energy level so initial cluster head selection is done randomly. And remaining node in that grid is joining with that cluster head.

Energy calculation: energy of each and every node going to reduce whenever nodes receiving or transferring or ideal mode.

Energy calculation formula is given bellow. 
Transmitting power $=$ txp " $\mathrm{w}$ "

Receiving power $=$ Rxp "w"

Initial energy = Ei “j”

Packet size $=$ Ps "Kb"

$\mathrm{Tx}$ data rate $=\mathrm{Dt}$ " $\mathrm{Mb} / \mathrm{s}$ "

$\mathrm{Rx}$ data rate $=\mathrm{Rt}$ "Mb/s"

Current energy = Ec “j”

Time duration for transfer and receive $=\mathrm{T}$ " $\mathrm{ms}$ "

Energy loss per Tx packet $=\left(\operatorname{txp}^{*} \mathrm{~T}\right) \mathrm{j}$

Energy loss per Rx packet $=\left(\operatorname{Rxp}^{*} \mathrm{~T}\right) \mathrm{j}$

Number of packet transferred per $\sec =>\mathrm{Npk}=\mathrm{Dt} / \mathrm{Ps}$ or $\mathrm{Dr} / \mathrm{Ps}$

Energy loss per second $=(\operatorname{txp} * \mathrm{~T}) * \mathrm{Npk}+(\mathrm{Rxp} * \mathrm{~T}) * \mathrm{Npk}$

$\mathrm{Ec}=\mathrm{Ec}-$ Energy loss “j”

\section{Cluster head changes:}

In sensor network, if we forming the clustering mean we can improve the network life time is possible but the constant cluster head mean that particular node will lose more energy. To avoid this problem we need to use cluster head changes. There is the different cluster head changing algorithm is available like as LEACH. In that algorithm cluster head changes will be in random manner, so that may chances to reduce the some particular node energy. To avoid this problem we are using the HEF algorithm.

Each and every second calculated energy is going to store in energy list, whenever cluster head duration is over then we are comparing energy of each and every node in that list. And which one having high energy in that list we are selecting that node as the cluster head for that period.

Block diagram Explanation:

In our project, we are considering only the communication part; we are not implementing any sensing part. The data unit is used to generate the data. Each and every node has the energy unit. Energy unit has the current energy level.

Routing manager controls the all events such as sending, receiving and data transmission. In base station, node has the node list and energy compassion unit and data unit. Data

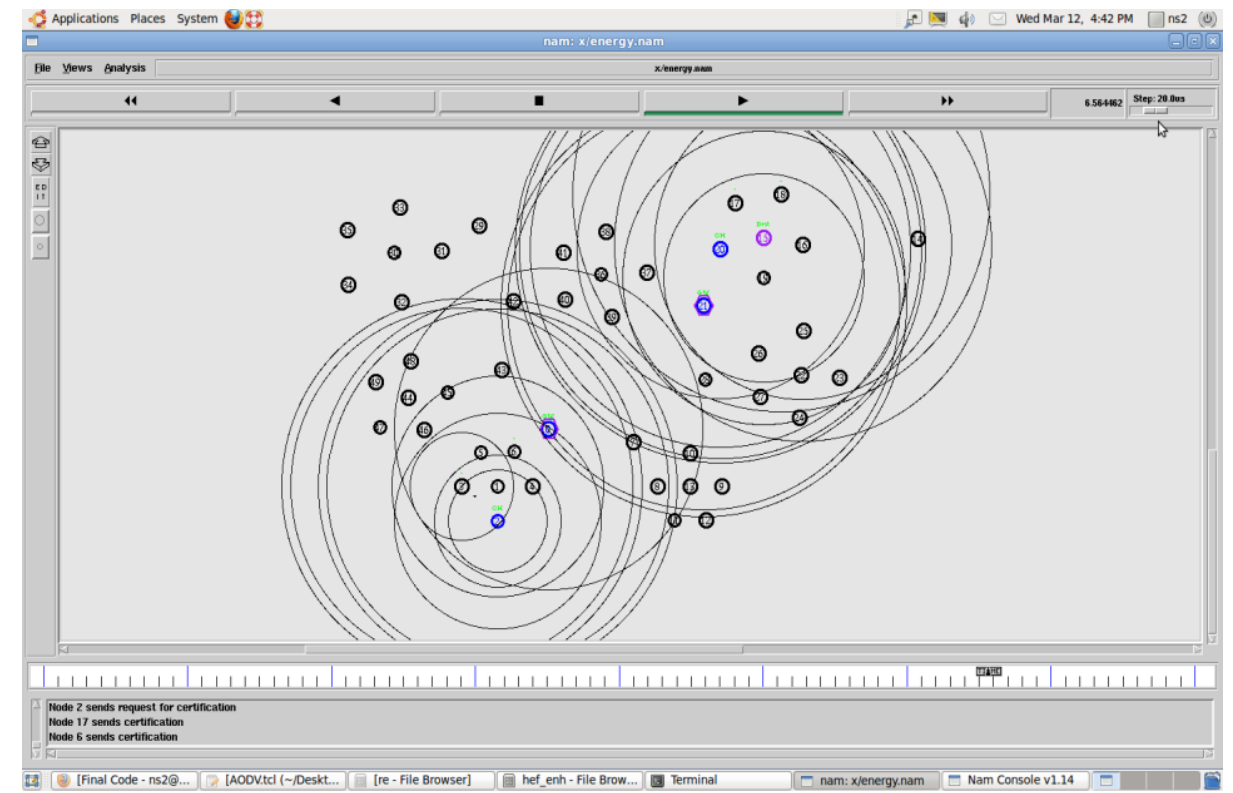

Fig 2. Network Animator showing Changes in Cluster Heads and Cluster Heads sending Packets to the destination.

Unit collects all info. Energy comparison unit compare the energy level and gives high remaining energy node name. Routing manger will selects $\mathrm{CH}$ by HEF. And timer used for trigger the event to compare the energy level. 


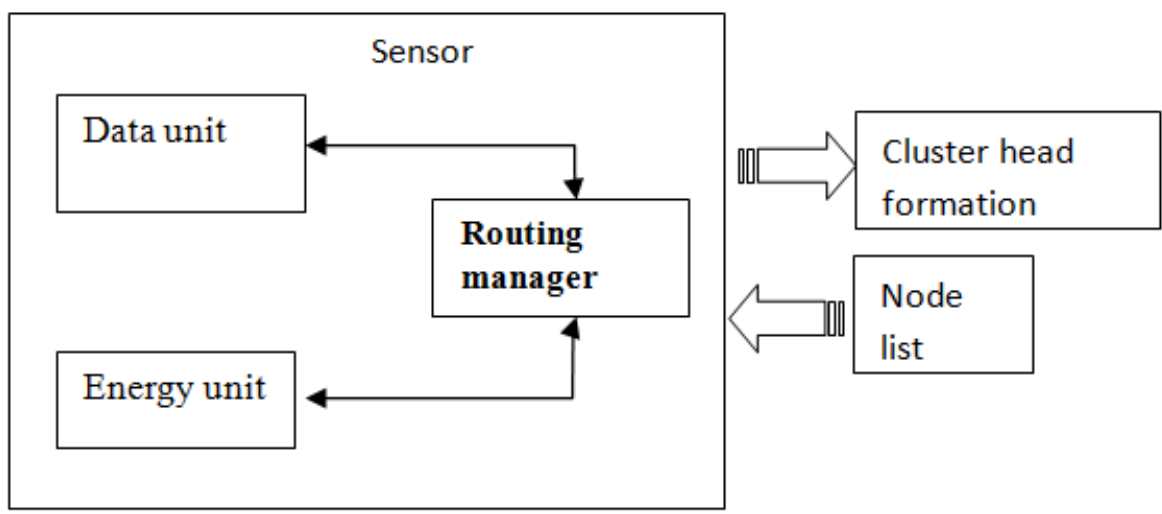

FIG 3. Block diagram for Cluster Head Formation.

IV. Secure Data Transmission:

The unreliable communication channel and unattended operation make the security defenses even harder. Indeed, as pointed out in wireless sensors often have the processing characteristics of machines that are decades old (or longer), and the industrial trend is to reduce the cost of wireless sensors while maintaining similar computing power. With that in mind, many researchers have begun to address the challenges of maximizing the processing capabilities and energy reserves of wireless sensor nodes while also securing them against attackers. All aspects of the wireless sensor network are being examined including secure and efficient routing, data aggregation, group formation, and so on. In addition to those traditional security issues, we observe that many general-purpose sensor network techniques assumed that all nodes are cooperative and trustworthy. This is not the case for most, or much of, real-world wireless sensor networking applications, which require a certain amount of trust in the application in order to maintain proper network functionality. To detect the malicious node we are going to use capability of cryptographic security. In addition, there are many attacks designed to exploit the unreliable communication channels and unattended operation of wireless sensor networks. Furthermore, due to the inherent unattended feature of wireless sensor networks, we argue that physical attacks to sensors play an important role in the operation of wireless sensor networks.

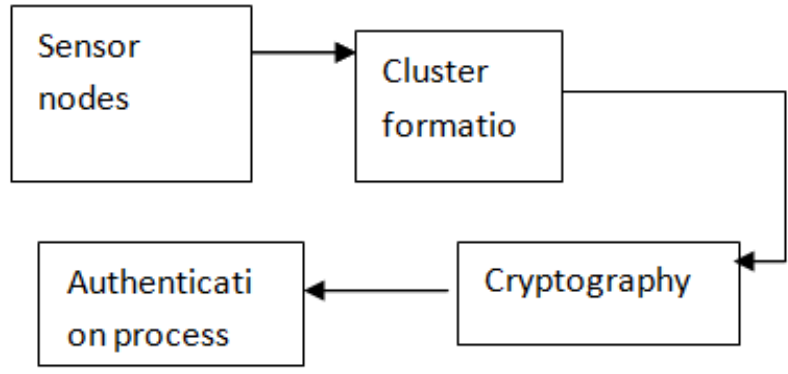

Fig 4.Block diagram for secured data transmission.

\section{Requirement Specification}

1. SOFTWARE REQURIEMENTS:

Tools: ns-allinone-2.34

Os: Linux

2. LANGUAGES:

Front End: TCL (Tool Command Language)

3. TECHNOLOGY USED:

Network Simulator-2 


\section{Results:}

Our project main aim is to increase life time of sensors, and assure the data security. So in our result we calculated minimum residual energy of the node in each grid.

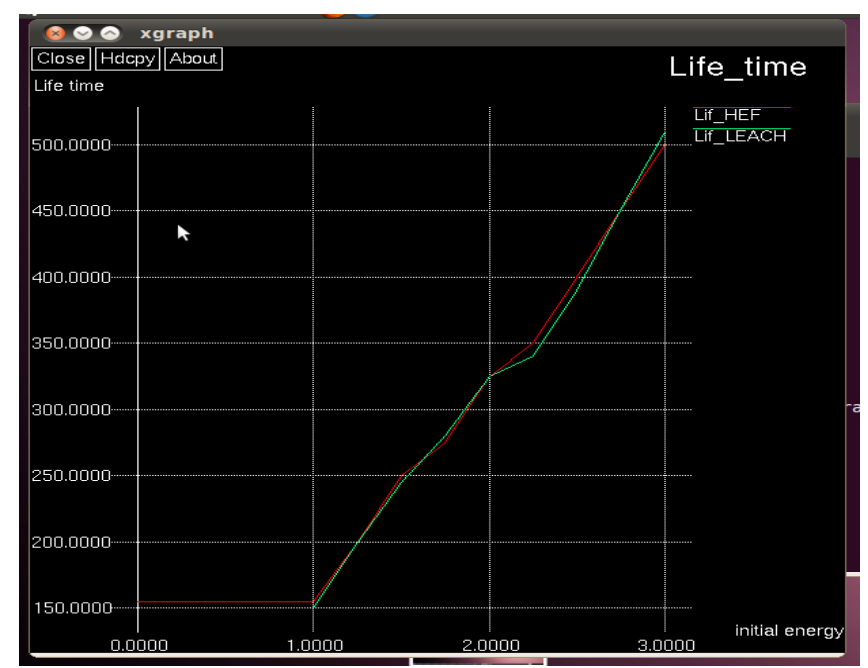

Fig 5. Graph showing comparison on lifetime of Leach and High Energy First Protocol.

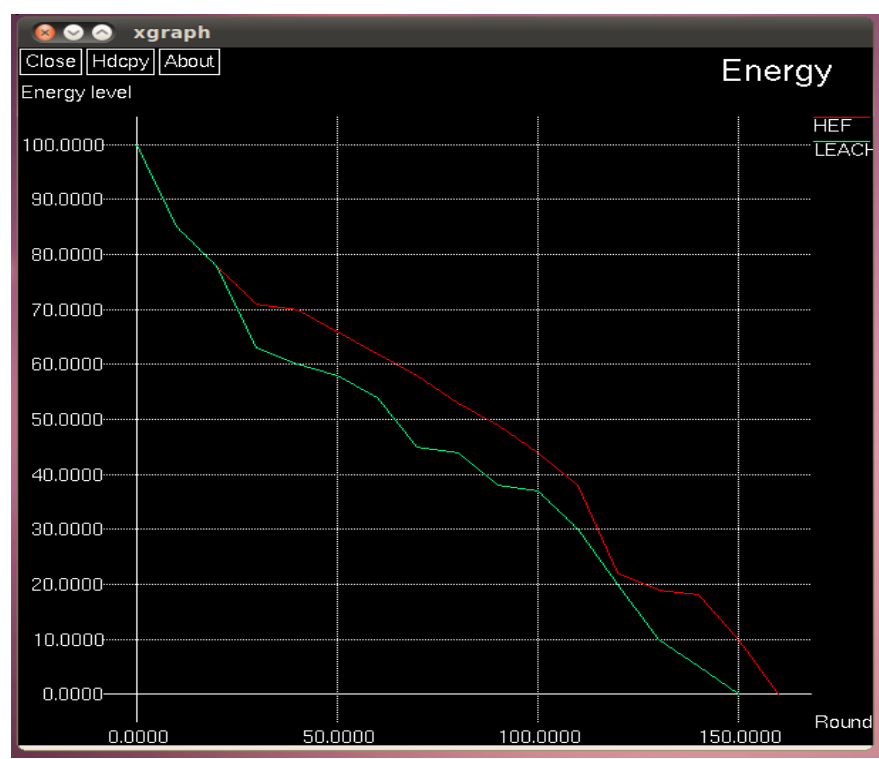

Fig 6 : Graph showing comparison on energy consumption between Leach and High Energy First Protocol

\section{Random cluster head selection}

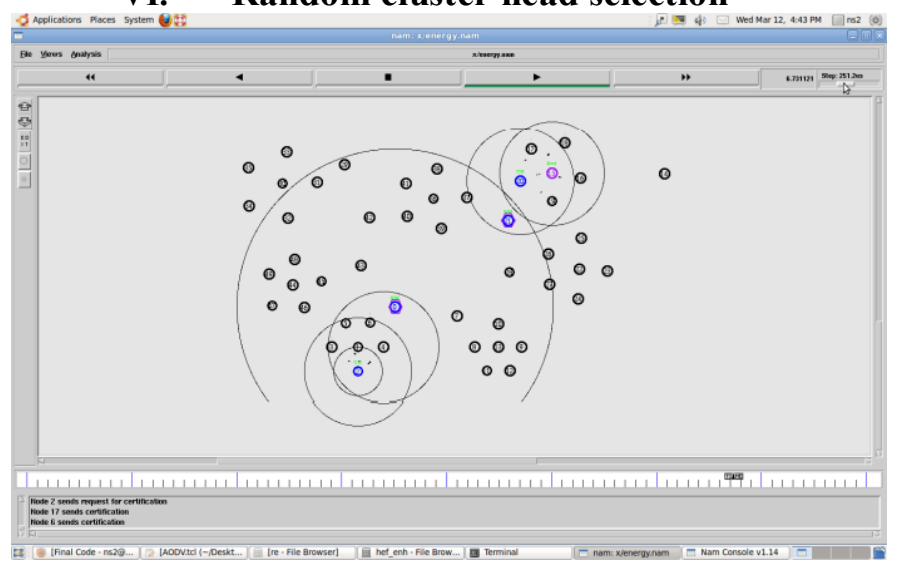

Fig 7.Fig showing Random Cluster Head selection. 


\section{Conclusion}

From our project we Conclude network life time increase when compare with previous method, in previous model there may be chances to repeated node may act as cluster head so energy loss of that certain node is high. But in our method we reduced the changes for repeated node as cluster head, in our model high energy node only going to act as $\mathrm{CH}$ so energy loss is less. In previous work, researchers not considered any security level with energy efficient routing but we included security scheme for sensor network to improve the transmission of data in a secured way.

\section{References:}

[1] W. Heinzelman, A. Chandrakasan and HBalakrishnan, "Energy-Efficient CommunicationProtocol for Wireless Micro sensor Networks",Proceedings of the 33rd Annual InternationalConference on System Sciences, Vol. 2, Maui, 4-7January 2002.

[2] M. Bani Yassein, A. Al-zou'bi, Y. Khamayseh,W. Mardini "Improvement on LEACH Protocol ofWireless Sensor Network"

[3] Bo Shen, et al, Cluster-Based Routing Protocolsfor Wireless Sensor Networks, Journal of Software,2006.

[4] Zhao Yulan, Jiang Chunfeng "Research aboutImprovement of LEACH Protocol" 2010

[5] Li Xunbo, Li Na, Chen Liang, Shen Yan, WangZhenlin, Zhu Zhibin “An Improved LEACH For Clustering Protocols In Wireless Sensor Networks",International Conference on Measuring Technologyand Mechatronics Automation,2010.

[6] Xu Long-long, Zhang Jian-jun, "ImprovedLEACH Cluster Head Multi-hops Algorithm inWireless Sensor Networks" Ninth InternationalSymposium on Distributed Computing and Applications to Business, Engineering andScience,2010.

[7] Mrs. V. Nithya (Asst. Professor), Shad drackYaw Nusenu (M.Tech Comm. Sys.), Dr. BRamachandran (Professor) a clustering protocolbased on tree routing algorithm in wireless sensor networks (wsn) International Conference onAdvanced Computing, Communication and Networks'11 1239

[8] Y. Liu, Y. Zhao and J. Gao, A New ClusteringMechanism Based On LEACH Protocol, inProceedings of the International Joint Conferenceon Artificial Intelligence, 2009. 